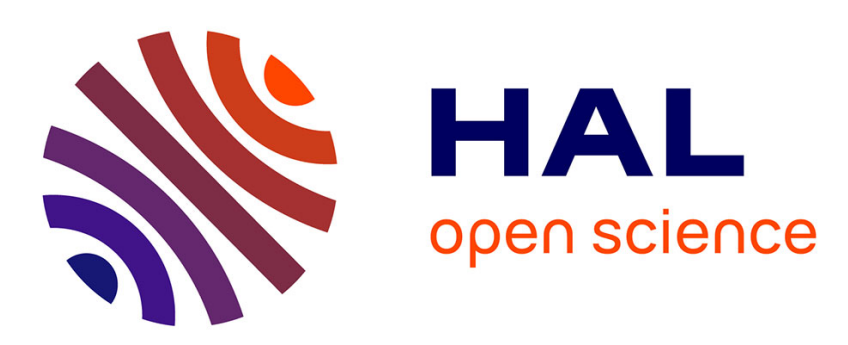

\title{
Thermal resistance of an interfacial molecular layer by first-principles molecular dynamics
}

Thuy-Quynh Duong, Carlo Massobrio, Guido Ori, Mauro Boero, Évelyne Martin

\section{- To cite this version:}

Thuy-Quynh Duong, Carlo Massobrio, Guido Ori, Mauro Boero, Évelyne Martin. Thermal resistance of an interfacial molecular layer by first-principles molecular dynamics. Journal of Chemical Physics, 2020, 153 (7), pp.074704. 10.1063/5.0014232 . hal-02990565

\section{HAL Id: hal-02990565 https://hal.science/hal-02990565}

Submitted on 17 Nov 2020

HAL is a multi-disciplinary open access archive for the deposit and dissemination of scientific research documents, whether they are published or not. The documents may come from teaching and research institutions in France or abroad, or from public or private research centers.
L'archive ouverte pluridisciplinaire HAL, est destinée au dépôt et à la diffusion de documents scientifiques de niveau recherche, publiés ou non, émanant des établissements d'enseignement et de recherche français ou étrangers, des laboratoires publics ou privés. 


\section{Thermal resistance of an interfacial molecular layer by first-principles molecular} dynamics

Thuy-Quynh Duong, ${ }^{1}$ Carlo Massobrio, ${ }^{2}$ Guido Ori, ${ }^{2}$ Mauro Boero, ${ }^{2}$ and Evelyne Martin $^{1, \text { a) }}$

${ }^{1)}$ Univ. Lille, CNRS, Centrale Lille, Yncréa ISEN, Univ. Polytechnique Hauts-de-France, UMR 8520 - IEMN, F-59000 Lille, France

${ }^{2)}$ Université de Strasbourg, CNRS, Institut de Physique et Chimie des Matériaux de Strasbourg, UMR 7504, F-67034 Strasbourg, France

(Dated: 29 June 2020)

The approach-to-equilibrium molecular dynamics (AEMD) methodology is applied in combination with first-principles molecular dynamics (FPMD) to investigate the thermal transfer between two silicon blocks connected by a molecular layer. Our configuration consists of alkanes molecules strongly coupled to the silicon surfaces via covalent bonds. In the phase 1 of AEMD, the two Si blocks are thermalized at high and low temperatures to form the hot and cold reservoirs. During the phase 2 of AEMD, a transfer between reservoirs occurs until thermal equilibrium is reached. The transfer across the interface dominates the transient over heat conduction within the reservoirs. The value of the thermal interface conductance is in agreement with experimental data obtained for analogous bonding cases between molecules and reservoirs. The dependence on length of the thermal interface resistance features two contributions. One is constant (the resistance at the silicon/molecule interface) while the other varies linearly with the length of the molecular chains (diffusive transport). The corresponding value of the thermal conductivity agrees well with experiments.

a)Electronic mail: evelyne.martin@iemn.fr 


\section{INTRODUCTION}

Predicting the thermal conductivity of real materials at the atomic scale requires a precise account of chemical bonding and the use of a manageable scheme to describe thermal transport. This goal has been achieved by combining the methodology inherent in the "approach-to-equilibrium" molecular dynamics (AEMD) to first-principles molecular dynamics (FPMD). AEMD is based on the production of a transient regime allowing for substantial reductions (factor of 10) in the computational cost with respect to alternative approaches ${ }^{1-4}$. The robustness of FPMD stems from the use of density functional theory to express the interatomic potential energy thereby leading to a self-consistent account of both the atomic and electronic degrees of freedom. The success of FPMD rests on a wealth of achievements in materials science for a range of properties going well beyond mere structural determination at finite temperatures ${ }^{5-7}$. We have recently shown that quantitative predictions can also be obtained for the thermal properties of glassy systems, by calculating the thermal conductivity of glassy $\mathrm{GeTe}_{4}\left(g-\mathrm{GeTe}_{4}\right)\left(\mathrm{AEMD}-\mathrm{FPMD}, 0.15 \mathrm{~W} \mathrm{~K}^{-1} \mathrm{~m}^{-18}\right.$ vs $0.14 \mathrm{~W} \mathrm{~K}^{-1} \mathrm{~m}^{-1}$, experiments $\left.{ }^{9}\right)$. The thermal transient occurred on a timescale fully accessible to FPMD for computational cells containing up to 645 atoms.

In this work we demonstrate that the combination of AEMD and FPMD can also be fruitfully employed to obtain realistic interface thermal conductances/resistances. Nowadays, (nanoscale) interfaces are essential components for materials and devices used in thermoelectric energy conversion, electrochemical energy storage and nanoelectronics. Thermal transport through interfaces with nanoscale gaps is of crucial importance for their performance ${ }^{10}$. However, interfaces can act as the heat transport bottlenecks since the characteristic dimensions of nanodevices approach electron and/or phonon mean free paths ${ }^{11,12}$. So far, classical molecular dynamics (CMD) based on interatomic potentials has been widely used to calculate thermal properties of interfaces. However, the strength of the interaction between atoms at the interface, qualitatively expressed within $\mathrm{CMD}$, strongly impacts the value of the thermal interface resistance, calling for a higher level of accuracy to be pursued to achieve quantitative assessments. For example in Ref. 13, the force between a molecular layer and gold is tuned from 1 to 5 , which results in a variation of the interfacial resistance from 15 to $60 \mathrm{MW} \mathrm{m} \mathrm{m}^{-2} \mathrm{~K}^{-1}$.

We reckon that FPMD is the method of choice whenever the details of chemical bonding at the interface cannot be simplified by avoiding the explicit account of the electronic structure.

In the present letter, we adopt the AEMD/FPMD strategy to model thermal transport in an in- 

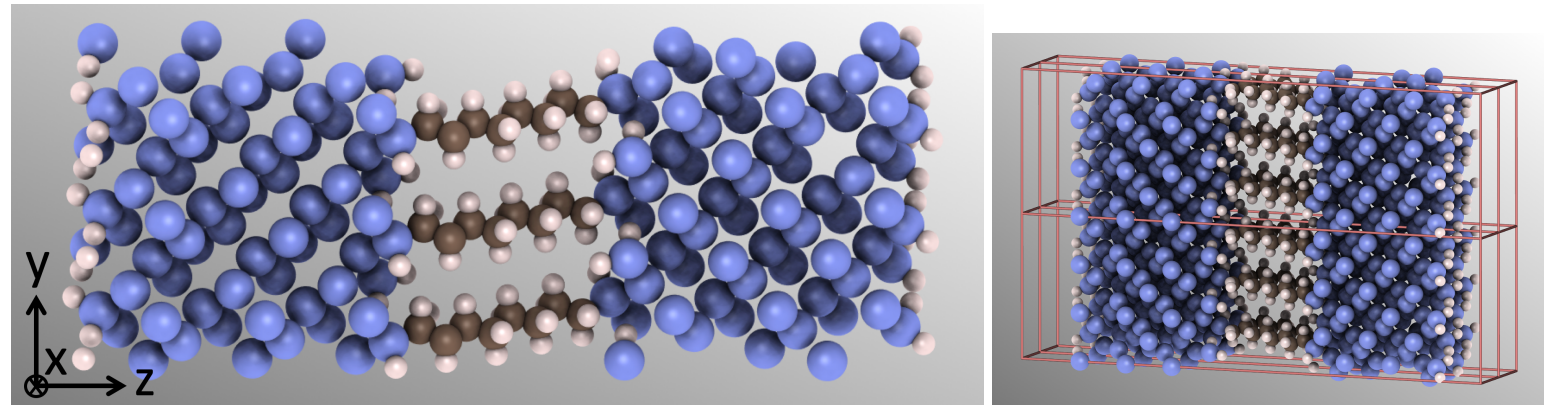

FIG. 1. Simulation cell (left) duplicated in directions $x$ and $y$ (right) to visualize the final structure formed of a layer of molecules sandwiched between two blocks of silicon. Si atoms are represented in blue, $\mathrm{C}$ in brown and $\mathrm{H}$ in white.

terfacial layer containing alkanes molecules. Because of their simple structure, alkane molecules are perfect candidates for AEMD/FPMD applications since they have been experimentally characterized in Ref. 14 as a function of length. The molecules are self-assembled, deposited on a gold surface and bonded via a thiol group. Their thermal conductance is measured by using a scanning thermal microscope (SThM) with a silicon tip. In the present study the molecules are sandwiched between two blocks of the same material, i.e. silicon, implying that covalent bonds are formed between the molecules and the Si blocks. In this way, the thermal transfer via the molecular layer is designed to be optimal and also tractable within the timescale of FPMD.

\section{MODELLING APPROACH}

We consider the atomic configuration shown in Fig. 1 (case of octanes). The blocks of silicon contain $N_{\mathrm{Si}}=72$ atoms each, corresponding to 3 cubic elementary cells in the $y\left(n_{y}=3\right)$ and $z$ directions $\left(n_{z}=3\right)$ and one unit cell in the $x$ direction $\left(n_{x}=1\right)$. The cross section ( $x y$ plane) is equal to $S=0.88 \mathrm{~nm}^{2}$. The Si internal and external surfaces are passivated by hydrogen atoms, except when a bond is form with one of the 3 molecules that connect the two blocks. The density of the molecules is equal to $3.4 \mathrm{~nm}^{-2}$, close to the experimental value of Ref. $14\left(4.6 \mathrm{~nm}^{-2}\right)$. While the simulation cell is duplicated in the three directions, the two blocks of Si do not interact with each other due to the inclusion of a void space (thickness of several $\mathrm{nm}$ ) in the $z$ direction (right part of Fig. 1).

The atomic structure is relaxed and thermalized to room temperature using FPMD ${ }^{15}$. The simulations are carried out with the CPMD code ${ }^{16}$. We use the exchange functional of Becke ${ }^{17}$ 
and the correlation part of Lee, Yang and Parr ${ }^{18}$ (BLYP). Norm-conserving pseudo-potentials ${ }^{19}$ are used to describe core-valence interactions. A plane-wave basis set is chosen with an energy cutoff of 50 Ry. The Brillouin zone integration is restricted to the $\Gamma$ point. The control of the ionic temperature is carried out by employing the Nosé-Hoover thermostat ${ }^{20,21}$. The integration step is 2.5 a.u. (0.06 fs) throughout our calculations and the fictitious electron mass is 300 a.u.

AEMD is implemented to study the thermal transfer via the molecular interfacial layer. During phase 1, thermostats are used to achieve a hot block on one side and a cold block on the other side of the molecular layer. We set the temperatures of the blocks to $T_{\mathrm{h}}=400 \mathrm{~K}$ (hot) and $T_{\mathrm{c}}=$ $200 \mathrm{~K}$ (cold), the evolution in time for the temperature of each block being plotted in Fig. 2. The target temperatures are reached in less than 1 ps but the thermostats are maintained up to 10 ps to stabilize the temperature profile along $z$. The temperature profile shown in Fig. 3 has a dominant

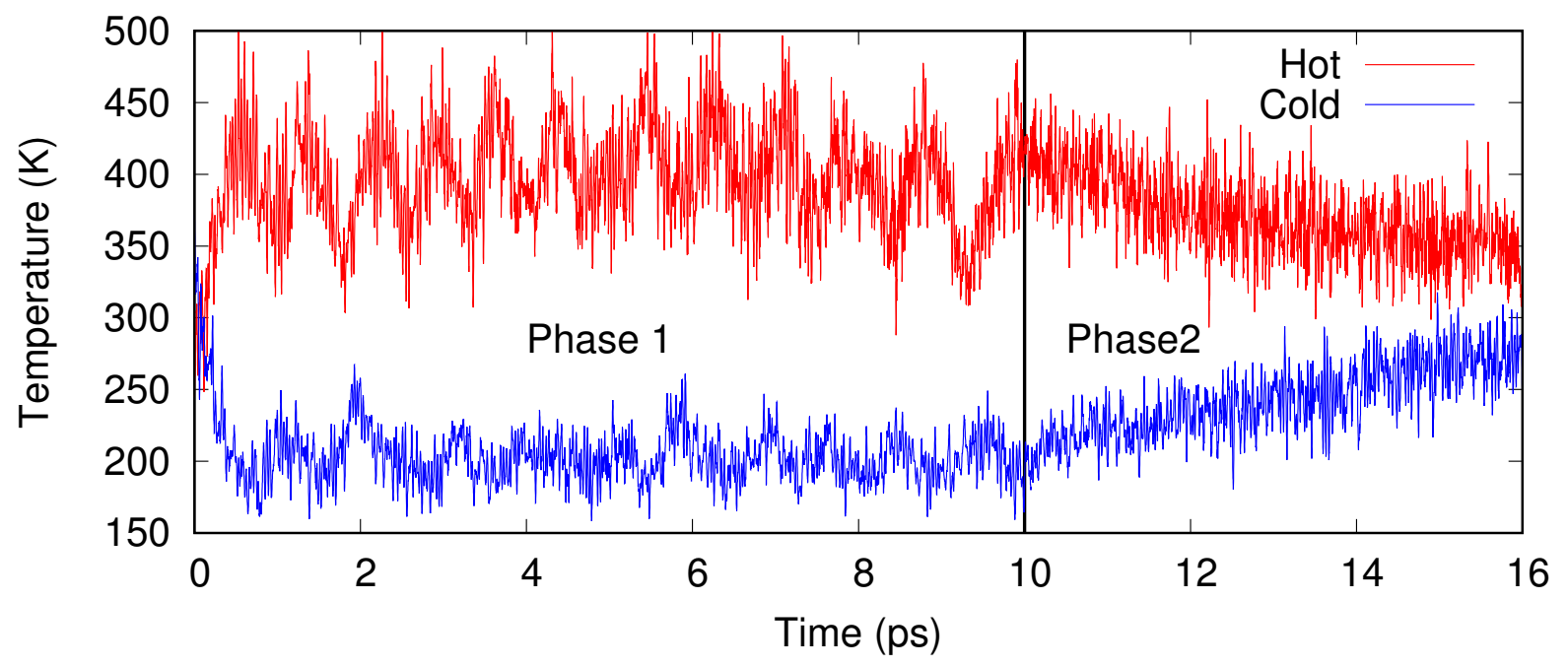

FIG. 2. Temperature in the hot and cold silicon blocks during phase 1 and 2. Case of octanes.

and linear gradient in the region where the molecules lie. The thermostats imposed to the silicon blocks are dropped at the end of phase 1, followed in phase 2 by an increase (decrease) of the temperature of the hot (cold) block (Fig. 2), the transient regime lasting 6 ps. The difference of temperature $\Delta T=T_{\mathrm{h}}-T_{\mathrm{c}}$ between the hot block (at $T_{\mathrm{h}}$ ) and the cold block (at $T_{\mathrm{c}}$ ) is plotted in Fig. 4. $\Delta T$ decreases exponentially with time as highlighted on the semi-logarithmic plot. The transient time $\tau$ defined as $\Delta T(t)=\Delta T_{0} \exp (-t / \tau)$ is equal to $6.6 \pm 0.1 \mathrm{ps}$. The behavior of the temperature profile for the phase 2, averaged on the interval [2-4] ps is given in Fig. 3. We notice that the temperature difference has decreased compared to phase 1 since substantial thermal transport through the interfacial layer has occurred. As during phase 1, the variation of the temperature 


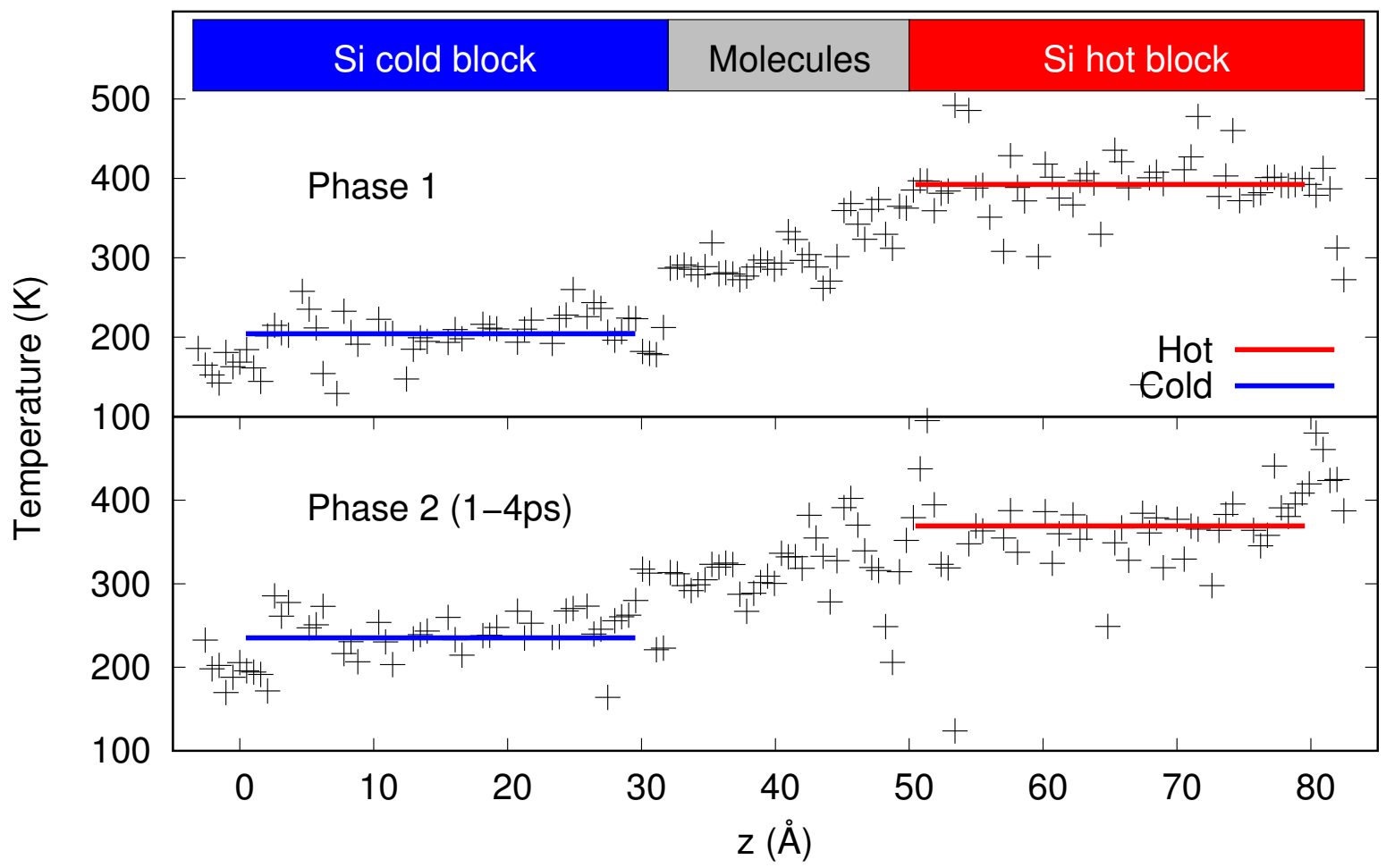

FIG. 3. Temperature profile averaged during the whole duration of phase 1 and during the interval $[1-4]$ ps of phase 2. Case of octanes.

is mainly located in the region where the molecules lie. These pieces of evidence show that $a$ ) the conduction is rapid within each block allowing for an homogeneous profile to set in and $b$ ) the transfer through the interface hampers the establishment of thermal equilibrium throughout the whole system (blocks plus interface) as schematized in Fig. 5. Therefore, we have a small resistance to conduction within each reservoir compared with the resistance to heat transfer between the two reservoirs. This physical situation is termed lumped capacitance approximation ${ }^{22}$.

The above rationale is used to analyze the thermal transient instead of solving the heat equation, as in the case of bulk materials ${ }^{1,8,23,24}$. Being very rapid within each block, the conduction in these parts does not contribute to the transient regime and it is negligible. As a consequence, the transient is governed by a balance between the rate of change of the internal energy of the reservoir (left hand side) and the rate of heat loss at the interface (right hand side):

$$
\begin{aligned}
& C \frac{d T_{\mathrm{h}}}{d t}=-G_{\text {Int }}\left(T_{\mathrm{h}}-T_{\mathrm{c}}\right), \\
& C \frac{d T_{\mathrm{c}}}{d t}=-G_{\text {Int }}\left(T_{\mathrm{c}}-T_{\mathrm{h}}\right) .
\end{aligned}
$$

with $C$ the heat capacity of the reservoir and $G_{\text {Int }}$ the conductance of the interface molecular layer 


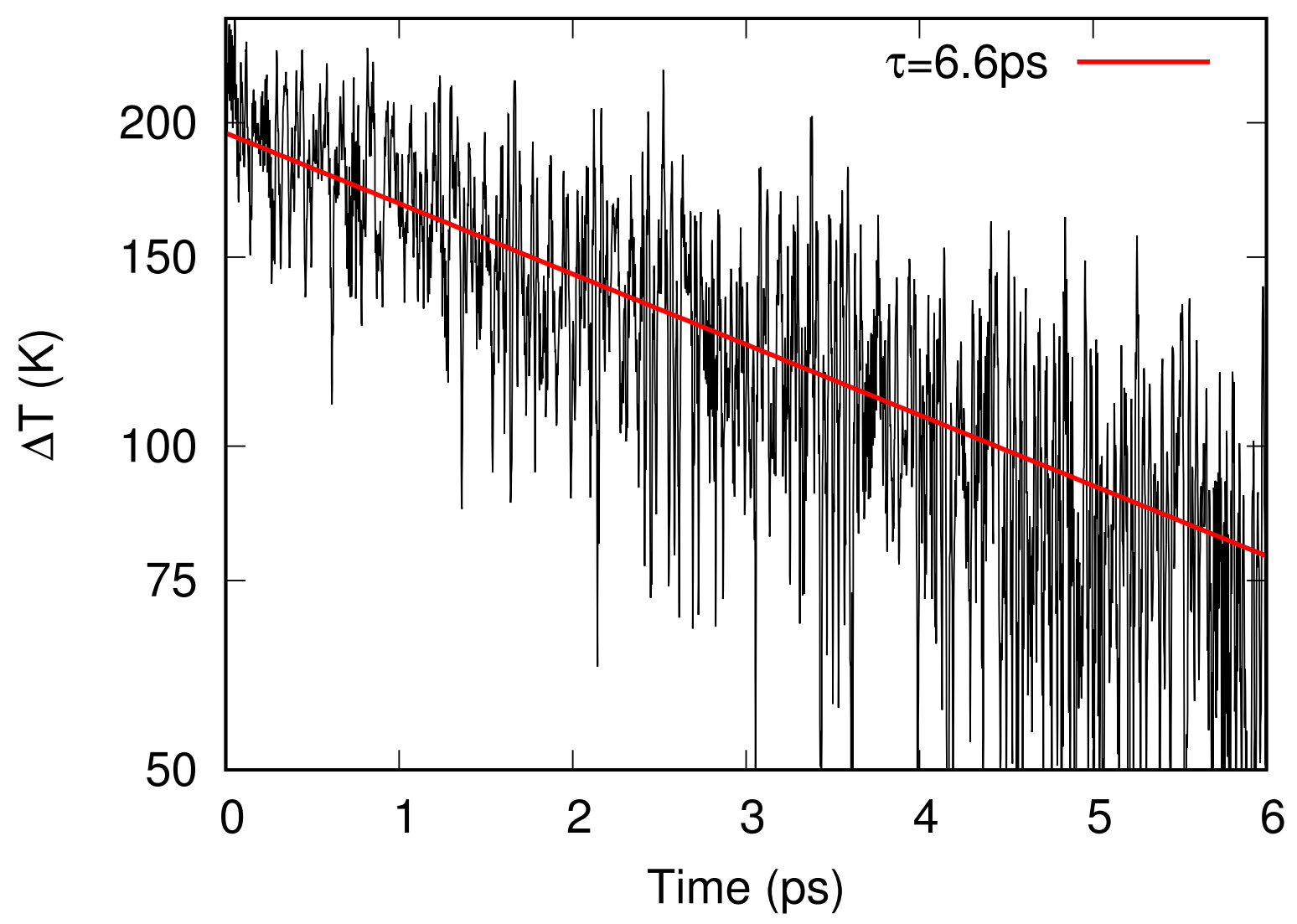

FIG. 4. Evolution of the temperature difference between the hot and cold silicon blocks during the phase 2 of the AEMD methodology. Case of octanes.

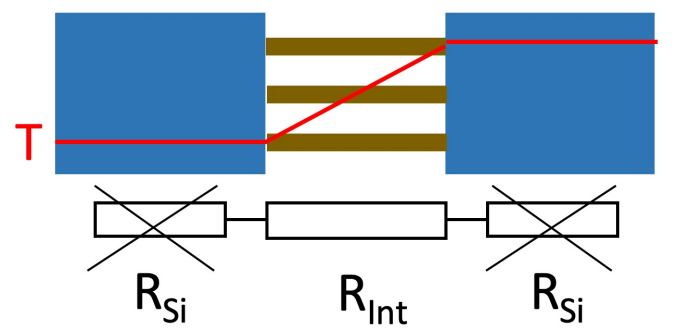

FIG. 5. Temperature profile in each Si block. The resistance of the blocks $R_{\mathrm{Si}}$ is negligible compared to the resistance of the interface $R_{\mathrm{Int}}$.

(the inverse of the interface resistance $R_{\text {Int }}$ ). The temperature difference $\Delta T$ obeys the equation:

$$
\frac{d \Delta T}{d t}=-\frac{2 G_{\text {Int }}}{C} \Delta T
$$

leading to $\Delta T=\Delta T_{0} \exp (-t / \tau)$ with $\tau=C / 2 G_{\text {Int }}$. The conductance of the molecular layer can be 
obtained from $\tau$ according to:

$$
G_{\text {Int }}=\frac{C}{2 \tau} .
$$

The heat capacity of a Si block $C$ is obtained from the variation of the total energy as a function of temperature and is equal to $3 N_{\mathrm{Si}} k_{\mathrm{B}}$ within an accuracy of $2 \%, N_{\mathrm{Si}}$ being the number of atoms in the hot/cold blocks respectively.

\section{RESULTS}

The resulting thermal conductance is plotted in Fig. 6. Calculations have been repeated for molecules containing 2 up to $16 \mathrm{C}$ atoms. The values are given in units of $\mathrm{MW} \mathrm{m} \mathrm{m}^{-2} \mathrm{~K}^{-1}$, that

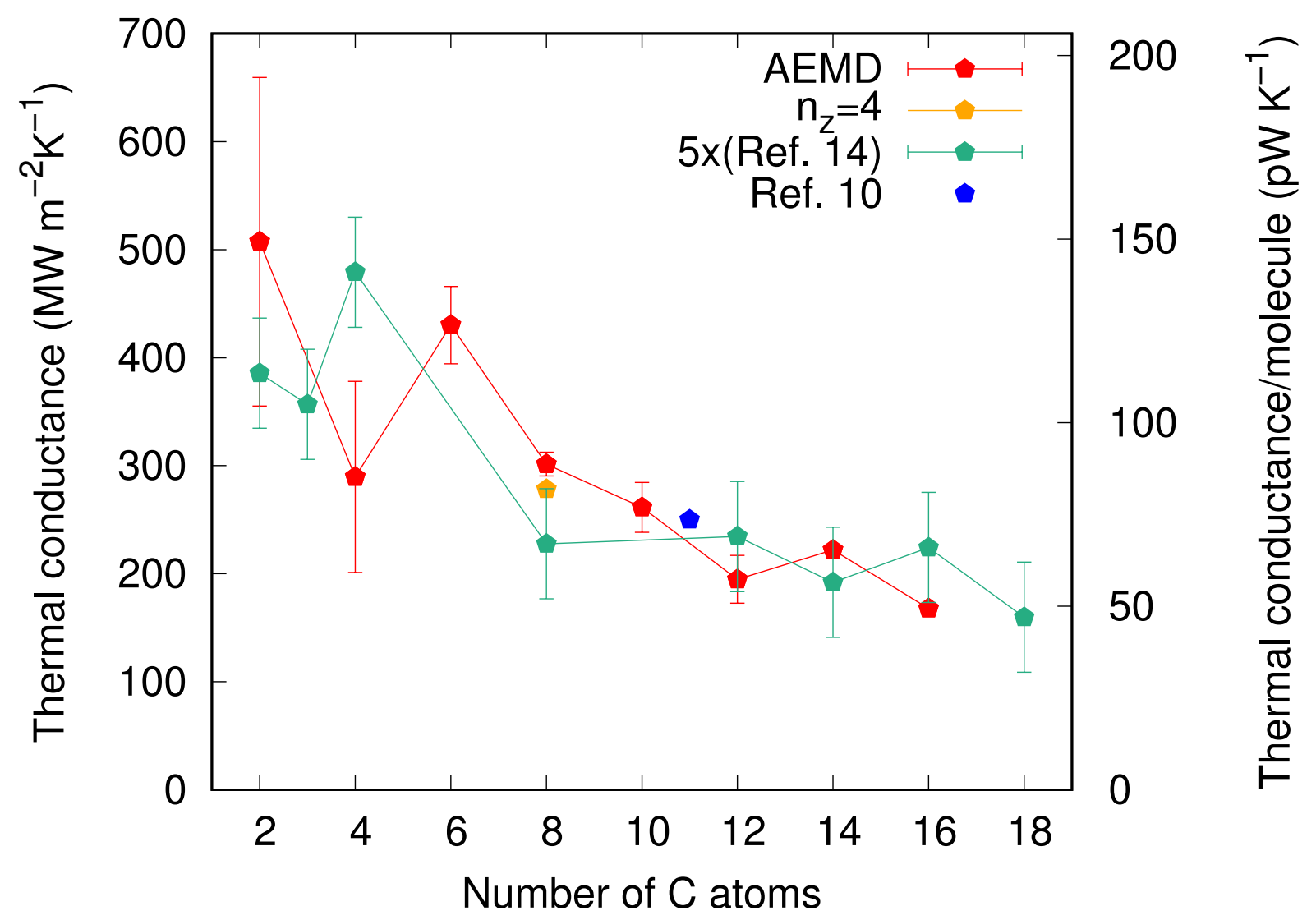

FIG. 6. Thermal conductance of the interface $G_{\text {Int }}$ as a function of the number $N$ of C per molecule obtained by AEMD for $n_{z}=3$ (red dots) and $n_{z}=4$ (orange dot). The green dots are the experiments of Ref. 14 multipled by a factor of 5 . The blue dot is the thermal conductance between reservoirs in diamond obtained in Ref. 10.

corresponds to $G_{\text {Int }} / S$ and in units of $\mathrm{pW} \mathrm{K}^{-1}$ per molecule, i.e. $G_{\text {Int }} / 3$. Each point is the average 
over several AEMD trajectories, displayed with standard deviations. We also performed a calculation with thicker blocks of silicon ( $n_{z}=4$, thickness of $2.2 \mathrm{~nm}$ instead of $n_{z}=3$, thickness of 1.6 $\mathrm{nm})$. The results are close by confirming that the thermal transfer depends mainly on the interface. Also, the resistance of each block is negligible even with $n_{z}=3$, the default value.

Analysis of Fig. 6 reveals that the thermal conductance of the interface is higher in comparison with the experiment of Ref. 14. Here we recall that alkanethiol molecules were studied in that work with a thiol group promoting respectively a $\mathrm{Au}-\mathrm{S}$ interfacial bond at the interface with $\mathrm{Au}$ and van der Waals interactions via a methyl group with the block of Si. In comparison, in our model we considered two strong $-\mathrm{CH}_{2}-\mathrm{Si}$ - covalent bonding interactions at both ends of the alkane molecules, this meaning that the overall interaction of the alkane molecule with the substrate is expected to be higher than in Ref. 14. This argument is supported on the basis of the strength of the relevant interactions: $-\mathrm{CH}_{2}-\mathrm{Si}-(\sim 89.6 \mathrm{kcal} / \mathrm{mol})>-\mathrm{S}-\mathrm{Au}-(\sim 60.6 \mathrm{kcal} / \mathrm{mol})>-\mathrm{CH}_{3} \cdots \mathrm{Si}$ $(0.5-1 \mathrm{kcal} / \mathrm{mol})^{25}$.

It appears that the strong $-\mathrm{CH}_{2}-\mathrm{Si}$ - covalent bonding to the $\mathrm{Si}$ blocks is at the very origin of the higher thermal conductance when compared to experiments. Indeed, at a quantitative level, the two results (FPMD/AEMD and experiments of Ref. 14) can be reconciled by amplifying the experimental data by a factor of 5, as we did in Fig. 6. This extrapolation is consistent with high levels of thermal conductance (380 to $600 \mathrm{MW} \mathrm{m}^{-2} \mathrm{~K}^{-1}$ ) recorded experimentally when the bonding between the reservoirs and molecules is tuned to facilitate the transfer at the interface ${ }^{11}$.

We have also plotted in Fig. 6 (blue point) another calculation performed with interatomic potentials ${ }^{10}$ for an alkane containing $11 \mathrm{C}$ covalently bonded to diamond reservoirs. This configuration consists of an interface linked to the reservoirs by $\mathrm{C}-\mathrm{C}$ bonds only $\left(-\mathrm{CH}_{2}-\mathrm{C}-: \sim 88.5\right.$ $\mathrm{kcal} / \mathrm{mol})^{25}$, thereby mitigating the qualitative character of interatomic potentials compared to the case of heterogeneous bonding. For comparable densities of molecules, the value found in Ref. 10 is in agreement with our results (see Fig. 6).

The question arises on the diffusive or ballistic nature of the heat transport in the system. For the Si blocks, this issue cannot be addressed since the transient is only sensitive to the transfer across the interface (lumped capacitance assumption). The situation changes for the molecules and it can be considered by looking at the dependence of the resistance with the length $l$ of the molecules or the number $N$ of $\mathrm{C}$ atoms. Provided the thermal transport is purely diffusive, the thermal resistance $R_{\text {Int }}$ should vary linearly with the length according to $l /(\kappa . S), \kappa$ being a thermal conductivity. $R_{\text {Int }}$ is represented as a function on $N$ in Fig. 7. The thermal resistance fluctuates around $3.10^{9} \mathrm{~K} \mathrm{~W}^{-1}$ 


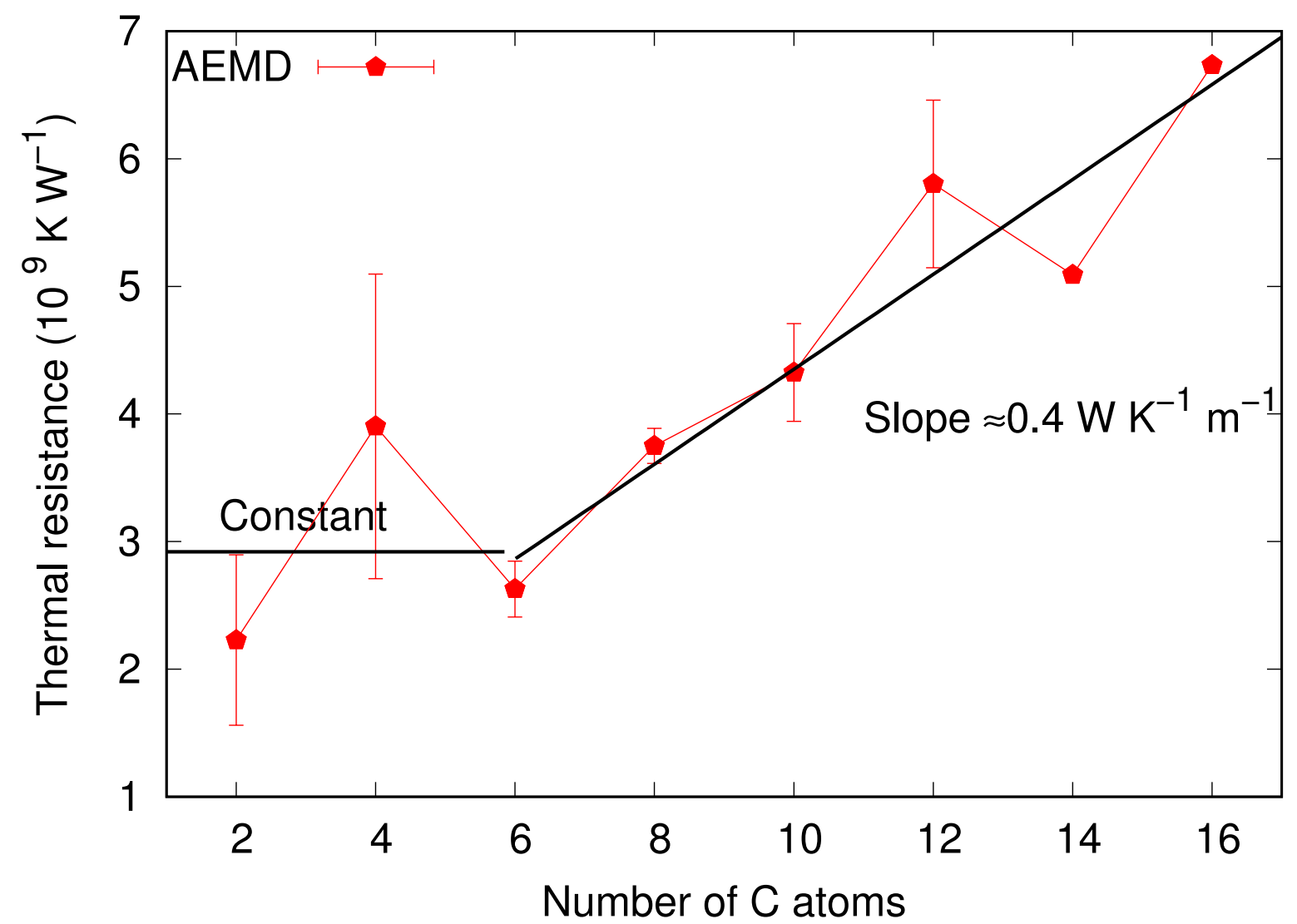

FIG. 7. Thermal resistance $R_{\mathrm{Int}}$ as a function of the number of $\mathrm{C}$ atoms per molecule.

for small $N$ to increase linearly with $N$ for $N$ larger than 8 , and can be written as :

$$
R_{\mathrm{Int}}(l)=2 R_{\mathrm{Si} / \mathrm{mol}}+R_{m o l}(l)
$$

Therefore, the thermal interface resistance $R_{\text {Int }}$ can be viewed as the sum of a constant term $R_{\mathrm{Si} / \mathrm{mol}}$ corresponding to the thermal resistances of $\mathrm{Si} /$ molecule contacts and a term $R_{\text {mol }}(l)$ varying linearly with $l$ that expresses the Fourier conduction along the molecules. The slope of the linear regime in Fig. 7 leads to a value of $\kappa=0.4 \mathrm{~W} \mathrm{~K}^{-1} \mathrm{~m}^{-1}$, commonly accepted for polyethylene (see for example Refs. 26-29).

\section{CONCLUSION}

In conclusion, we have applied the approach-to-equilibrium (AEMD) methodology to study the thermal transfer across a molecular layer by resorting to first-principles molecular dynamics (FPMD). The analysis of the temperature profile showed that the thermal transient inherent in 
phase 2 of AEMD is dominated by the transfer via the molecular layer. The corresponding decay time gives access to a thermal conductance and its inverse, a thermal resistance. The thermal conductance takes values recorded experimentally when bonding is tuned to facilitate the transfer at the interface. It also turns out that the thermal conductance is 5 times larger than other experimental data obtained using a scanning thermal microscope and featuring weaker bonds between one end of the molecular chain and the reservoir.

The dependence of the thermal interface resistance on the length of the molecules is indicative of a double contribution. The first is constant, not depending on the length of the molecules and corresponding to the $\mathrm{Si} /$ molecules interface. The second is proportional to the length of the molecule following a Fourier regime. From this second contribution we are able to extract a thermal conductivity in good agreement with analogous molecular species.

\section{ACKNOWLEDGMENTS}

G.O. acknowledges the Seed Money program of Eucor-The European Campus (project MEDIA) for financial support. This work was funded by the French ANR via the project n. ANR-17CE09-0039-02 "SIRENA". Calculations were performed by using resources from GENCI (Grand Equipement National de Calcul Intensif) (Grant No. x2016095071, A0030910296, A0040905071, A0050910296), Pôle HPC Equip@ Meso of the University of Strasbourg, and clustphy2 at the IEMN.

The data that support the findings of this study are available from the corresponding author upon reasonable request.

\section{REFERENCES}

${ }^{1}$ E. Lampin, P. L. Palla, P.-A. Francioso, and F. Cleri, "Thermal conductivity from approach-toequilibrium molecular dynamics," Journal of Applied Physics 114, 033525 (2013).

${ }^{2}$ H. Zaoui, P. L. Palla, F. Cleri, and E. Lampin, "Length dependence of thermal conductivity by approach-to-equilibrium molecular dynamics," Physical Review B 94, 054304 (2016).

${ }^{3}$ A. Bouzid, H. Zaoui, P. L. Palla, G. Ori, M. Boero, C. Massobrio, F. Cleri, and E. Lampin, "Thermal conductivity of glassy $\mathrm{GeTe}_{4}$ by first-principles molecular dynamics," Physical Chemistry Chemical Physics 19, 9729-9732 (2017). 
${ }^{4}$ P. L. Palla, S. Zampa, E. Martin, and F. Cleri, "Interface thermal behavior in nanomaterials by thermal grating relaxation," International Journal of Heat and Mass Transfer 131, 932-943 (2019).

${ }^{5}$ D. Marx and J. Hutter, Ab Initio Molecular Dynamics: Basic Theory and Advanced Methods (Cambridge University Press, 2009).

${ }^{6}$ G. Ori, A. Bouzid, E. Martin, C. Massobrio, S. Le Roux, and M. Boero, “Chalcogenide glasses as a playground for the application of first-principles molecular dynamics to disordered materials," Solid State Sciences 95, 105925 (2019).

${ }^{7}$ C. Massobrio, M. Boero, S. L. Roux, G. Ori, A. Bouzid, and E. Martin, "Making Computer Materials Real: The Predictive Power of First-Principles Molecular Dynamics," in Theory and Simulation in Physics for Materials Applications: Cutting-Edge Techniques in Theoretical and Computational Materials Science, Springer Series in Materials Science, edited by E. V. Levchenko, Y. J. Dappe, and G. Ori (Springer International Publishing, Cham, 2020) pp. 3-21.

${ }^{8}$ T.-Q. Duong, C. Massobrio, G. Ori, M. Boero, and E. Martin, "Thermal conductivity and transport modes in glassy $\mathrm{GeTe}_{4}$ by first-principles molecular dynamics," Physical Review Materials 3, 105401 (2019).

${ }^{9}$ S.-N. Zhang, J. He, T.-J. Zhu, X.-B. Zhao, and T. M. Tritt, “Thermal conductivity and specific heat of bulk amorphous chalcogenides $\mathrm{Ge}_{20} \mathrm{Te}_{80-x} \mathrm{Se}_{x}(x=0,1,2,8)$," Journal of Non-Crystalline Solids 355, 79-83 (2009).

${ }^{10}$ Y. Wang, Y. Cao, K. Zhou, and Z. Xu, “Assessment of Self-Assembled Monolayers as HighPerformance Thermal Interface Materials,” Advanced Materials Interfaces 4, 1700355 (2017).

${ }^{11}$ P. J. O’Brien, S. Shenogin, J. Liu, P. K. Chow, D. Laurencin, P. H. Mutin, M. Yamaguchi, P. Keblinski, and G. Ramanath, "Bonding-induced thermal conductance enhancement at inorganic heterointerfaces using nanomolecular monolayers," Nature Materials 12, 118-122 (2013).

${ }^{12}$ H. Han, Y. Zhang, N. Wang, M. K. Samani, Y. Ni, Z. Y. Mijbil, M. Edwards, S. Xiong, K. Sääskilahti, M. Murugesan, Y. Fu, L. Ye, H. Sadeghi, S. Bailey, Y. A. Kosevich, C. J. Lambert, J. Liu, and S. Volz, "Functionalization mediates heat transport in graphene nanoflakes," Nature Communications 7, 1-9 (2016).

${ }^{13}$ L. Hu, L. Zhang, M. Hu, J.-S. Wang, B. Li, and P. Keblinski, "Phonon interference at selfassembled monolayer interfaces: Molecular dynamics simulations," Physical Review B 81, 235427 (2010).

${ }^{14}$ T. Meier, F. Menges, P. Nirmalraj, H. Hölscher, H. Riel, and B. Gotsmann, 'Length-Dependent 
Thermal Transport along Molecular Chains,” Physical Review Letters 113, 060801 (2014).

${ }^{15}$ R. Car and M. Parrinello, "Unified Approach for Molecular Dynamics and Density-Functional Theory," Physical Review Letters 55, 2471-2474 (1985).

${ }^{16}$ See http://www.cpmd.org, copyright 2000-2019 jointly by IBM Corporation and by Max Planck Institute, Stuttgart.

${ }^{17}$ A. D. Becke, "Density-functional exchange-energy approximation with correct asymptotic behavior," Physical Review A 38, 3098-3100 (1988).

${ }^{18}$ C. Lee, W. Yang, and R. G. Parr, "Development of the Colle-Salvetti correlation-energy formula into a functional of the electron density," Physical Review B 37, 785-789 (1988).

${ }^{19}$ N. Troullier and J. L. Martins, "Efficient pseudopotentials for plane-wave calculations," Physical Review B 43, 1993-2006 (1991).

${ }^{20}$ S. Nosé, “A molecular dynamics method for simulations in the canonical ensemble," Molecular Physics 52, 255-268 (1984).

${ }^{21}$ W. G. Hoover, “Canonical dynamics: Equilibrium phase-space distributions,” Physical Review A 31, 1695-1697 (1985).

${ }^{22}$ T. L. Bergman, F. P. Incropera, D. P. DeWitt, and A. S. Lavine, Fundamentals of Heat and Mass Transfer, seventh ed. (John Wiley \& Sons, 2011).

${ }^{23}$ H. Zaoui, P. L. Palla, F. Cleri, and E. Lampin, "Fourier-like conduction and finite onedimensional thermal conductivity in long silicon nanowires by approach-to-equilibrium molecular dynamics," Physical Review B 95, 104309 (2017).

${ }^{24}$ H. Zaoui, P. L. Palla, S. Giordano, F. Cleri, M. Verdier, D. Lacroix, J.-F. Robillard, K. Termentzidis, and E. Martin, “Thermal conductivity of deca-nanometric patterned Si membranes by multiscale simulations," International Journal of Heat and Mass Transfer 126, 830-835 (2018).

${ }^{25}$ W. M. Haynes, CRC Handbook of Chemistry and Physics: A Ready-Reference Book of Chemical and Physical Data (CRC Press, Boca Raton, FL, 2014) oCLC: 892709696.

${ }^{26}$ I. Krupa, I. Novák, and I. Chodák, "Electrically and thermally conductive polyethylene/graphite composites and their mechanical properties," Synthetic Metals 145, 245-252 (2004).

${ }^{27}$ C.-M. Ye, B.-Q. Shentu, and Z.-X. Weng, "Thermal conductivity of high density polyethylene filled with graphite,” Journal of Applied Polymer Science 101, 3806-3810 (2006).

${ }^{28}$ J. Gu, Q. Zhang, J. Dang, J. Zhang, and Z. Yang, “Thermal conductivity and mechanical properties of aluminum nitride filled linear low-density polyethylene composites," Polymer Engineering \& Science 49, 1030-1034 (2009). 
${ }^{29}$ J. Yu, B. Sundqvist, B. Tonpheng, and O. Andersson, “Thermal conductivity of highly crystallized polyethylene," Polymer 55, 195-200 (2014). 